\title{
Entrelacs
}

Cinéma et audiovisuel

12 | 2016

Nouvelles formes audiovisuelles documentaires

\section{Des nœuds dans le Web : la commémoration du 17 octobre 1961 sur Internet.}

Philippe Brand

\section{OpenEdition}

\section{Journals}

\section{Electronic version}

URL: http://journals.openedition.org/entrelacs/1874

DOI: 10.4000 /entrelacs. 1874

ISSN: 2261-5482

\section{Publisher}

Éditions Téraèdre

\section{Electronic reference}

Philippe Brand, «Des nœuds dans le Web : la commémoration du 17 octobre 1961 sur Internet. », Entrelacs [Online], 12 | 2016, Online since 14 January 2016, connection on 30 April 2019. URL : http:// journals.openedition.org/entrelacs/1874; DOI : 10.4000/entrelacs.1874

This text was automatically generated on 30 April 2019

Tous droits réservés 


\title{
Des nœuds dans le Web : la commémoration du 17 octobre 1961 sur Internet.
}

\author{
Philippe Brand
}

1 L'héritage de la Guerre d'Algérie est devenu le plus récent "passé qui ne passe pas," pour emprunter le titre célèbre de l'étude des années Vichy écrite par Éric Conan et Henry Rousso ${ }^{1}$. Plusieurs controverses ont éclaté en France autour de la commémoration du cinquantième anniversaire de la fin de la guerre, montrant jusqu'à quel point cette histoire reste irrésolue et perturbante. Un événement en particulier - le massacre des manifestants algériens à Paris le 17 octobre 1961 - est devenu une métonymie pour les pires excès de l'époque. En 2011, lors du cinquantième anniversaire de ces événements, deux webdocumentaires - La Nuit oubliée $e^{2}$ et $17.10 .61^{3}$ - ont paru sur Internet avec le but d'éclaircir ce moment historique pour les nouvelles générations. Cet article analyse les stratégies esthétiques et narratives employées par ces deux webdocumentaires en quête de nouvelles manières de représenter une histoire longtemps cachée, naviguant entre ce que Henry Rousso décrit dans La Dernière catastrophe comme, "la tension, parfois l'opposition entre l'histoire et la mémoire, entre la connaissance et l'expérience, entre la distance et la proximité, entre l'objectivité et la subjectivité, entre le chercheur et le témoin ${ }^{4} »$.

2 Visant un public large, les deux webdocumentaires partagent un nombre de caractéristiques, comme une carte interactive de Paris, des clips vidéo de témoignages, des documents et des images des archives historiques, ainsi que des entretiens avec des historiens illustres. Le plan ouvert et non linéaire des deux webdocumentaires permet au spectateur d'explorer les circonstances autour du massacre de plusieurs perspectives. Selon ses envies, le spectateur pourrait adopter une approche spatiale à travers la navigation de la carte, ou il pourrait explorer les événements à partir d'un tableau chronologique. Confronté par la multitude de divers éléments contenus dans les webdocumentaires, le spectateur est forcé à jouer un rôle plus actif, devenant une espèce 
de chercheur ou détective qui doit rassembler les différents fragments narratifs pour créer un ensemble cohérent. Dans ce sens-là, les deux œuvres ressemblent à deux romans qui ont joué un rôle central dans la révélation initiale de ces événements à un public plus large : Meurtres pour mémoire de Didier Daeninckx ${ }^{5}$ et La Seine était rouge : Paris, octobre 1961 de Leïla Sebbar ${ }^{6}$. Le polar de Daeninckx raconte les recherches d'un détective qui découvre la vérité du massacre, tandis que le roman de Sebbar met l'accent sur l'importance des témoignages. Les deux webdocumentaires combinent ces approches narratives, dans le but d'aider les jeunes à démêler les fils complexes de ces événementsun exemple de ce que le critique Michael Rothberg décrit non comme un "lieu de mémoire », selon la formule célèbre de Pierre Nora, mais plutôt un «nœud de mémoire ${ }^{7}$ . \

\section{«Pour ne pas oublier »}

3 Cette phrase, écrite en majuscules rouges sur la page d'accueil de La Nuit oubliée, pourrait servir de raison d'être pour les deux webdocumentaires. La notion de l'oubli et la transmission de la mémoire sont centrales à la mission explicitement pédagogique des deux œuvres. De nombreux critiques et historiens ont écrit des études détaillées sur la censure des informations et la suppression de la mémoire après le 17 octobre (et la Guerre d'Algérie plus généralement), suivi par un processus de reconnaissance des événements lent mais progressif. Cette reconnaissance devient de plus en plus évidente dans la sphère publique, surtout autour des moments comme les trentième, quarantième, et cinquantième anniversaires des événements. Deux éléments de ce processus sont particulièrement pertinents pour les deux webdocumentaires : la notion de « devoir de mémoire ", intensifiée ici par les associations complexes entre le 17 octobre et les années Vichy, le rôle du témoin et la tension entre la mémoire et l'histoire.

Des historiens ont en effet souligné des liens complexes entre les mémoires de la Guerre d'Algérie et les années Vichy. Selon Henry Rousso :

On peut identifier en premier lieu une même tendance à la valorisation du souvenir doublée d'une condamnation irrévocable de toute forme d'oubli, une des grandes caractéristiques du régime d'historicité de la fin du XXe siècle. La notion de "devoir de mémoire" a ainsi imprégné toute la réflexion sur la guerre d'Algérie, comme elle l'a fait au même moment pour les souvenirs de Vichy et du nazisme ${ }^{8}$.

6 Si un « devoir de mémoire » est souvent invoqué dans une variété de contextes dans la société contemporaine, il est essentiel de noter que «se souvenir » est parfois bien plus compliqué qu'on ne le pense. Comme la mémoire individuelle, la mémoire collective peut être fragmentaire et sélective. De manière paradoxale, malgré - ou peut-être à cause de - son occultation initiale, le 17 octobre a fini par supporter une toile dense d'associations interconnectées. Pour Michel Laronde, le 17 octobre est devenu «l'événement le plus référencé et référentiel des rapports conflictuels entre la France et l'Algérie sur le territoire national français ${ }^{9} »$. Henry Rousso va encore plus loin, affirmant que «Le "devoir de mémoire" [...] s'est cristallisé autour du souvenir du 17 octobre, devenu [...] une sorte de métaphore métonymique de cette guerre ${ }^{10} »$. Michael Rothberg décrit ce phénomène comme un exemple $d u$ "multidirectional memory ${ }^{11}$ ", où de multiples groupes d'acteurs apportent leurs passés traumatiques dans un présent changeant et hétérogène ${ }^{12}$. Les deux webdocumentaires illustrent le caractère 
multidirectionnel du 17 octobre à travers leurs discussions sur le rôle de Maurice Papon et leurs représentations du paysage mémoriel de la société française contemporaine.

7 Pour mieux comprendre les enjeux des deux webdocumentaires, il faut aussi considérer l'importance du témoignage et la tension entre mémoire et histoire. En parlant de la Shoah et de la Guerre d'Algérie, Rousso avance que « dans les deux cas, la prise de parole publique et la multiplication des témoignages ont joué un rôle essentiel, participant également du même régime d'historicité: "l'ère du témoin" va de pair avec l'ère de la mémoire ${ }^{13}$ ». Ici, Rousso cite le titre d'un livre important d'Annette Wieviorka, l'Ère du témoin, dans lequel elle trace l'importance grandissante du témoignage dans le XXe siècle. Wieviorka note que les historiens ont souvent manifesté " beaucoup de méfiance " envers les témoignages, les considérant comme remplis d'erreurs ou de possibles malentendus ${ }^{14}$. Or, dans un cas comme le 17 octobre, caractérisé par le silence de l'État et l'inaccessibilité des archives, il est peu surprenant que les témoins aient joué un rôle essentiel dans la transmission des événements. Rousso rappelle que les événements du 17 octobre sont constamment évoqués dans le cadre plus large de la Guerre d'Algérie, et il note que :

8 La mémoire du conflit joue toujours un rôle essentiel dans les relations entre la France et l'Algérie, et, en France, les polémiques continuent d'opposer les "vainqueurs" et les "vaincus", les partisans du FLN et de l'Algérie française, les contempteurs ou les défenseurs de l'armée, les harkis et les autres Algériens ${ }^{15}$.

Les deux webdocumentaires essaient d'évoquer les complexités de la situation dans leurs sélections des témoignages, qui représentent une variété de différents acteurs touchés par les événements.

\section{Les modèles narratifs}

Comme mentionné précedemment, La Nuit oubliée et 17.10.61 font écho aux thèmes et aux structures narratives des romans Meurtres pour mémoire de Didier Daeninckx, et La Seine était rouge : Paris, octobre 1961 de Leilla Sebbar. L'importance des archives est manifeste dans Meurtres pour mémoire où Roger Thiraud et son fils Bernard sont assassinés quand ils commencent à puiser dans des archives de la Deuxième Guerre mondiale. Rothberg note que le roman « joins a police thriller built on mystery, detection, and revelation with a plot of intergenerational historical transmission. [...] [The] point of Meurtres-the mystery that it stages in order to resolve-is the connection between different eras and the persistence of the unresolved past in the present ${ }^{16} »$. Le roman suit les conventions narratives traditionnelles d'un polar, et il présente l'Inspecteur Cadin comme un " détective-historien » dont l'enquête le mène aux archives et qui finit par révéler une vérité historique longtemps cachée et occultée par l'État ${ }^{17}$. Les deux webdocumentaires placent le spectateur dans une situation analogue. Le spectateur doit parcourir les différentes parties du site web pour reconstruire les éléments divers selon un récit cohérent. Le roman de Sebbar diffère notamment par le fait que l'accent est mis sur les témoignages - et les silences - individuels dans le moment présent, au lieu des recherches dans les archives historiques. Une mémoire collective longtemps tue revient à la surface quand Louis filme des témoignages pour son documentaire sur le 17 octobre, tandis qu'Amel et Omer parcourent la ville de Paris, retraçant les chemins des manifestants algériens trente ans plus tard. 
11 Anne Donadey soutient que les trois personnages principaux - Amel, Louis et Omer représentent les trois groupes principaux, « immigrés, Français, Algériens ${ }^{18}$ ", qui doivent confronter le 17 octobre et les héritages de la Guerre d'Algérie. Donadey remarque que « le fait que l'anamnèse dans le contexte post-colonial soit un processus délicat est souligné par les désaccords entre les trois personnages en ce qui concerne leur interprétation des événements et leurs divers choix de commémoration ${ }^{19}{ }$. Les témoignages que Louis rassemble ajoutent à la diversité des perspectives, car il filme une gamme diverse de participants et de témoins - un harki, un policier, des manifestants algériens, et plusieurs sympathisants et passants français. Enfin, il est important de noter que Sebbar n'essaie pas d'avancer une version définitive des événements. Comme Jonathan Lewis le remarque :

Sebbar does not proceed to straightforwardly present the reader with a singular, monolithic version of history to resolve the lack of historical transmission regarding 17 October 1961 and Algerian War. On the contrary, the ensuing narrative is inevitably fragmented, made up of various pieces of information collected from different sources, and constantly shifting from Amel's quest to retrace the footsteps of the Algerian demonstrators in the company of Omer to the description of clips from Louis's film ${ }^{20}$.

12 Cette esthétique fragmentaire et mobile est employée avec beaucoup de succès dans les deux webdocumentaires, qui se prêtent à une telle approche. Dans les deux cas, le spectateur doit trouver son propre chemin pour naviguer entre les différentes sections du site, et jouer un rôle actif pour interpréter les entretiens, les représentations fictives, et les matériaux des archives contenus sur le site.

\section{La Nuit oubliée}

13 Hébergé sur le site web du journal Le Monde, La Nuit oubliée a été créée par le journaliste Olivier Lambert et le photographe Thomas Salva. Le webdocumentaire propose des témoignages filmés qui sont illustrés par des photos et des séquences provenant d'archives historiques telles que l'INA. L'image de fond de la page d'accueil du site est une photo de pavés, sur laquelle est superposée une fenêtre cliquable pour lancer les différents témoignages filmés.

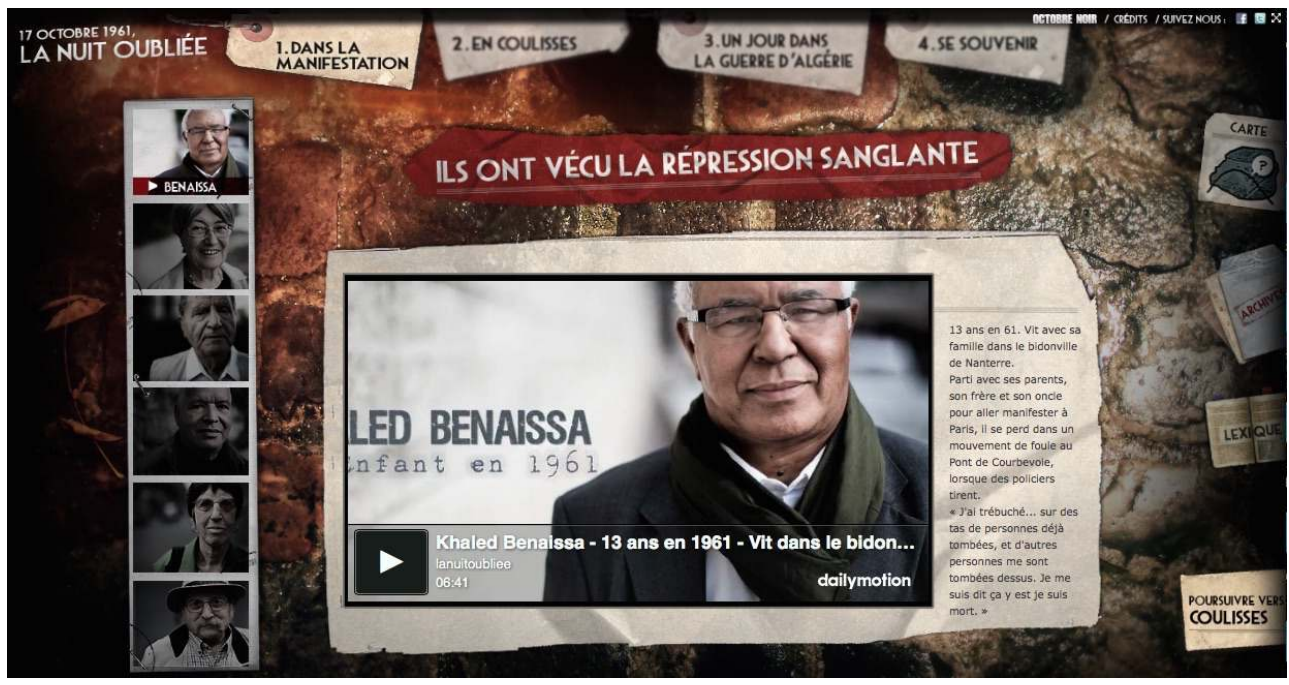

Figure 1 : La Nuit oubliée. Page d'accueil. (@ Olivier Lambert et Thomas Salva) 
14 À gauche se trouvent six photos des témoins sur lesquelles le spectateur peut cliquer pour sélectionner le témoignage voulu. En haut de la page, quatre icônes permettent la navigation entre les différentes parties du site: "Dans la manifestation», "En coulisses », « Un jour dans la Guerre d'Algérie », et " Se souvenir ». La première fois qu'un spectateur accède à chaque différente partie du webdocumentaire, une fenêtre apparaît en overlay avec une image tirée de la bande dessinée Octobre noir créée par Didier Daeninckx et Mako pour commémorer le cinquantième anniversaire du 17 octobre ${ }^{21}$. Octobre noir a un objectif explicitement pédagogique, avec une préface écrite par l'historien Benjamin Stora, et les créateurs de La Nuit oubliée emploient des images de la bande dessinée comme un fil conducteur entre les différentes parties, comme ils l'indiquent sur la première fenêtre en overlay qui accueille le spectateur en accédant au webdocumentaire : «À chaque page, les personnages de la BD Octobre noir vous guident ${ }^{22}$ $\gg$.

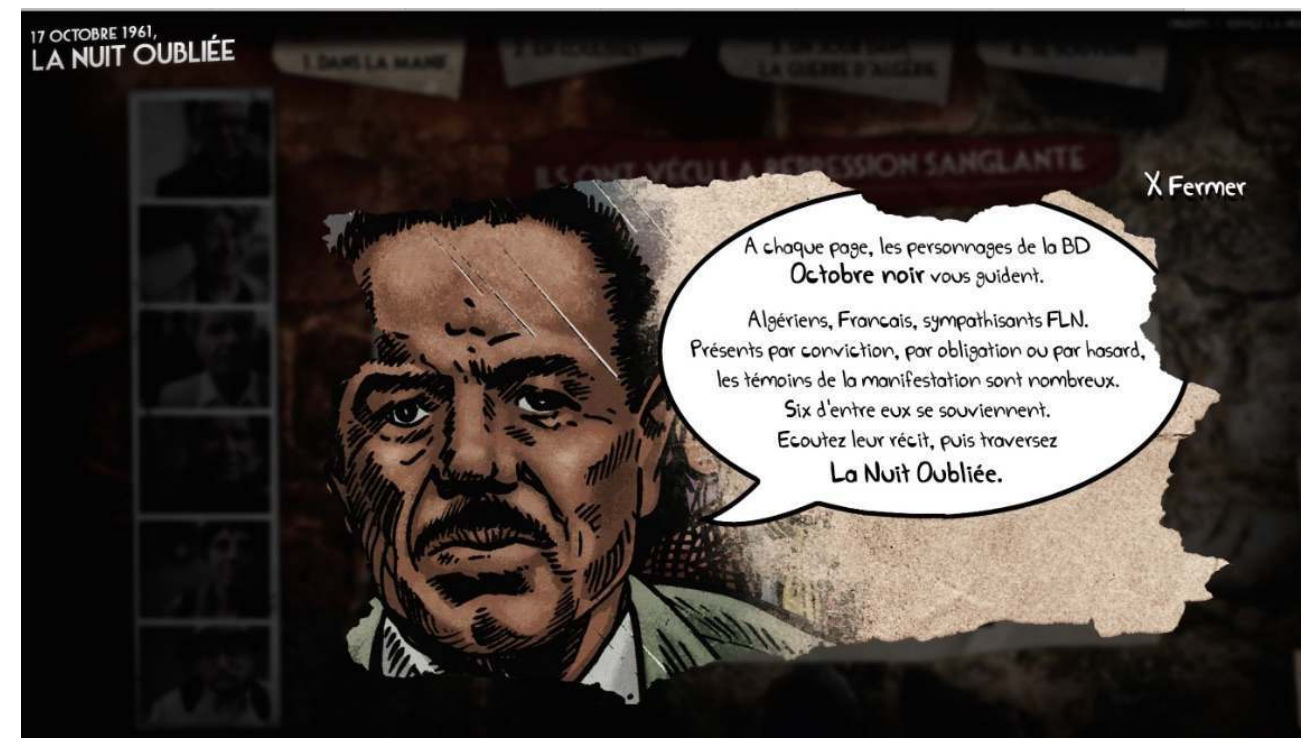

Figure 2 : La Nuit oubliée. Fenêtre en overlay. (@ Olivier Lambert et Thomas Salva)

Ce choix esthétique et narratif peut être compris de plusieurs façons. D'une part, cela suggère une crainte de la part des créateurs, comme s'ils pensaient que le spectateur pourrait se perdre dans l'interface du webdocumentaire sans un fil conducteur pour guider la navigation. Cela montre que même si l'on parle beaucoup de l'interactivité des webdocumentaires et la liberté d'un monde ouvert, cette liberté est souvent plus imaginaire que réelle. Nous allons voir que tandis que l'autre webdocumentaire, 17.10.61, privilégie l'idée de la déambulation, La Nuit oubliée encourage une lecture plutôt linéaire de ses éléments morcelés. L'autre considération capitale dans le choix d'une esthétique de bande dessinée est son attrait potentiel pour de jeunes générations. À la fin de sa préface, Stora affirme que «cette bande dessinée, forte, émouvante et érudite participe de la transmission mémorielle ${ }^{23} »$. Cette affirmation est remarquable dans son double appel aux émotions («émouvante ») et à l'intellect (" érudite »). Selon Wieviorka, un " appel à l'émotion ", véhiculé par l'insistance particulière sur des témoignages des victimes, et opposé "au caractère supposé "froid" de l'histoire écrite à partir des archives ", est devenu un des éléments centraux de l'histoire du XXe siècle ${ }^{24}$. Wieviorka cite les mémoires de Gideon Hausner, l'ordonnateur principal du procès d'Eichmann :

Le seul moyen de faire toucher du doigt la vérité était d'appeler les survivants à la

barre [...] et de demander à chacun un menu fragment de ce qu'il avait vu et de ce 
qu'il avait vécu. Le récit d'un certain enchaînement de circonstances fait par un seul témoin est suffisamment tangible pour être visualisé. Mises bout à bout, les dépositions successives de gens dissemblables, ayant vécu des expériences différentes, donneraient une image suffisamment éloquente pour être enregistrée. Ainsi espérais-je donner au fantôme du passé une dimension de plus, celle du réel ${ }^{25}$.

16 Un tel appel aux émotions par la mise en valeur des détails concrets des témoignages est évident dans La Nuit oubliée. Le webdocumentaire est plus complexe qu'une simple série d'entretiens et le format multimédia offre des capacités de créer « une dimension du réel » bien au-delà de ce que Gideon Hausner aurait pu imaginer dans les années 1960 . Lambert et Salva essaient de rendre tangibles les événements du 17 octobre avec un assemblage divers de matériaux : des photos et des clips vidéos des monuments parisiens d'aujourd'hui ; des photos, des enregistrements sonores, des documents et des séquences d'archives; des images de la bande dessinée Octobre noir; et des entretiens filmés avec des témoins et des historiens. À certains moments dans le webdocumentaire, plusieurs de ces éléments hétérogènes sont combinés, créant un effet de "mashup ». Cette esthétique pourrait être un appel aux jeunes générations, comme l'incorporation de la bande dessinée. En même temps, l'amalgame de ces éléments hétérogènes dans les témoignages filmés soulève plusieurs problèmes potentiels.

La première image que le spectateur voit dans le webdocumentaire est un visage d'un personnage d'Octobre noir, avec un court texte d'introduction signé par Lambert et Salva. Le premier mot, écrit en majuscules rouges dans un corps plus grand, est « OUBLI », suivi par les phrases :

18 Mais aussi censure, dissimulation ou déni. [...] 50 ans après, nous tentons ici de reconstruire une parcelle de cette histoire commune. POUR NE PAS OUBLIER. Et éviter de reproduire les erreurs du passé. ${ }^{26}$

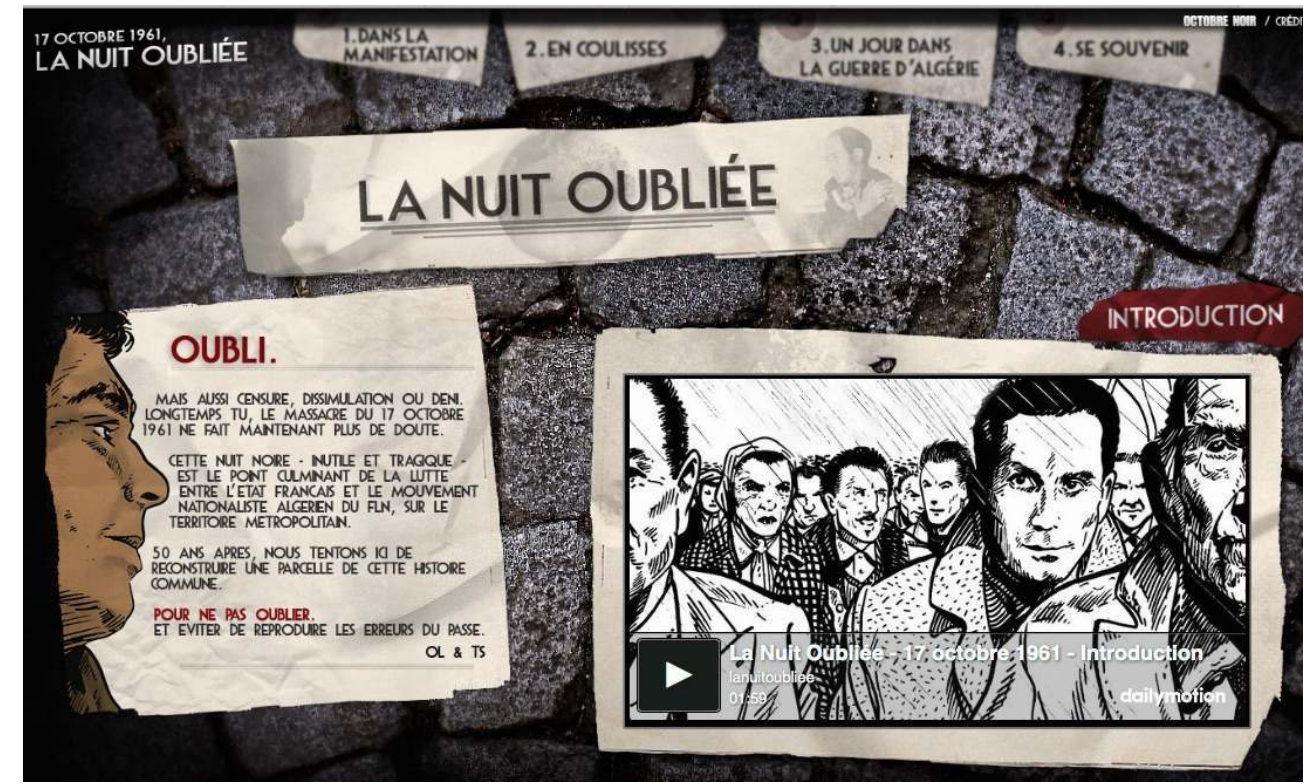

Figure 3 : La Nuit oubliée. Page d'accueil. (@ Olivier Lambert et Thomas Salva)

La description du 17 octobre comme « cette histoire commune » fait écho à un argument élaboré par Rothberg dans son analyse de Meurtres pour mémoire. Il soutient que le roman formule une revendication éthique : «by virtue of the accident of birth, national (and other) subjects inherit the imperative to investigate the multiple forms of violence that have been perpetrated in their name ${ }^{27} »$. Le texte de Lambert et Salva exprime une 
demande apparentée - le spectateur devrait non seulement enquêter sur la violence commise dans le passé, il devrait aussi agir dans le présent, pour éviter de commettre les mêmes erreurs. Au premier coup d'œil, cette demande paraît éminemment louable. Pourtant, la complexité extrême du 17 octobre le rend difficile pour le spectateur de savoir précisément de quelles erreurs les auteurs parlent. Pour un événement multidirectionnel comme le 17 octobre, caractérisé par ses liens avec le colonialisme, l'héritage des années Vichy, la violence de l'État, et le silence individuel et collectif, il est difficile de dire exactement quelles « erreurs du passé » il ne faut pas « reproduire ».

Les témoignages des victimes et des participants apparaissent dans une section du webdocumentaire intitulée "Dans la manifestation", sous une bannière rouge qui annonce "Ils ont vécu la répression sanglante ». Cette bannière sert à établir l'autorité des témoins qui apparaissent, et c'est aussi une référence à une plaque commémorative inaugurée par Bertrand Delanoë, le maire de Paris, en 2001. Cette plaque, inscrite avec la phrase « À la mémoire des nombreux Algériens tués lors de la sanglante répression de la manifestation pacifique du 17 octobre 1961 », a été le premier acte de commémoration officiel des événements du 17 octobre, et la plaque a déclenché des réactions vives ${ }^{28}$. Des images fragmentaires de cette plaque apparaissent brièvement dans l'entretien avec Khaled Benaissa, le premier témoin à parler dans le webdocumentaire, soulignant la nécessité de commémorer le 17 octobre de manière plus visible et durable.

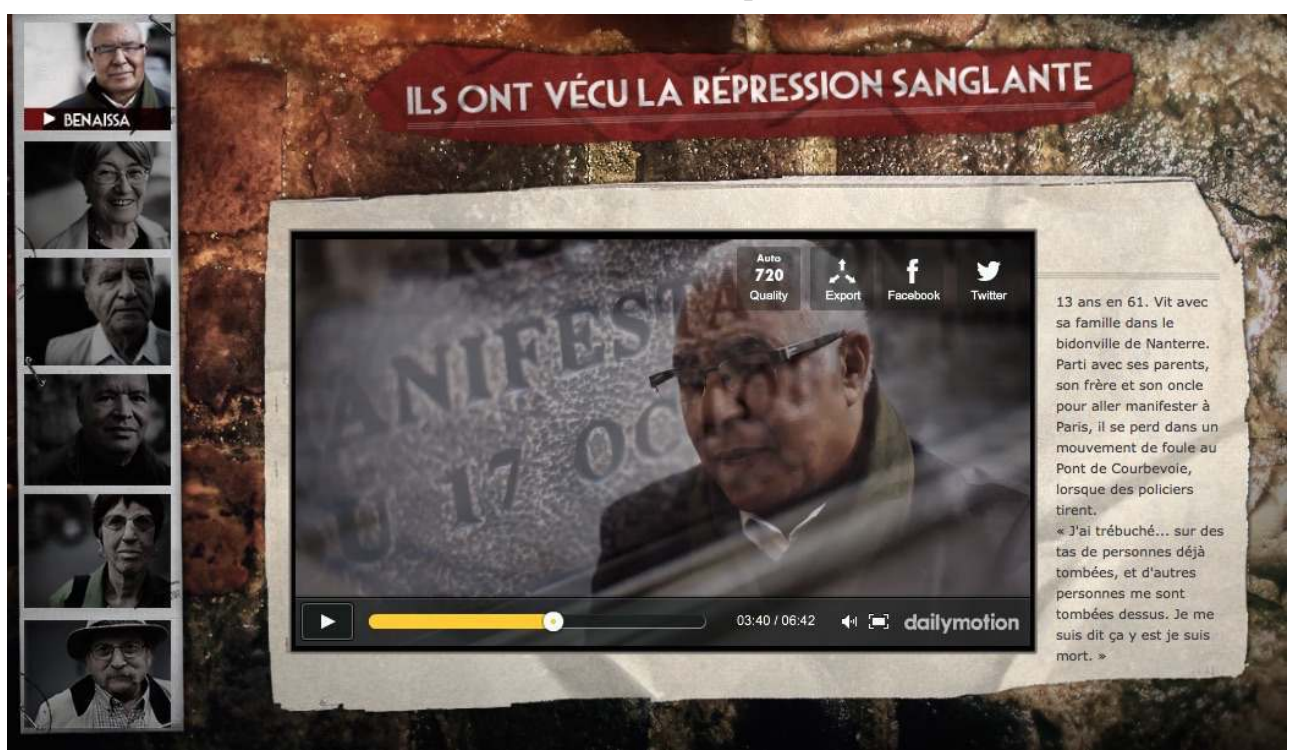

Figure 4 : La Nuit oubliée. Témoignage de Khaled Benaissa. (๔ Olivier Lambert et Thomas Salva)

Comme l'espace est limité et que tous les témoignages partagent le même format, je vais me concentrer sur les aspects des témoignages les plus exemplaires des questions en jeu dans le webdocumentaire. Chaque témoignage commence avec une petite phrase du témoin, filmé en plan rapproché, suivi par un bref montage de photographies et de vidéos, juxtaposant des images de 1961 avec des scènes de Paris aujourd'hui. Dans chaque entretien, le spectateur écoute la voix du témoin pendant que les images oscillent entre le présent et le passé, alternant entre l'entretien filmé en 2011 et des photos et des séquences d'archives. Or, il est important de noter que même si l'apparence de ces photographies et ces séquences suggère une origine dans les archives, la provenance des images n'est pas claire. Certaines de ces images sont clairement des photographies célèbres du 17 octobre, prises par les photographes Élie Kagan et Georges Azenstarck ${ }^{29}$, 
mais la plupart des images sont d'origine inconnue, ou du moins non déclarée. Les noms de Kagan et Azenstarck figurent sur la page « crédits » du webdocumentaire, avec l'INA et la BDIC-MHC ${ }^{30}$, mais dans l'ensemble, il y a très peu de documentation pour un webdocumentaire qui se veut pédagogique. En général, l'esthétique «mashup» réussit son but de créer une impression d'immédiateté, et la juxtaposition du passé et du présent renforce l'idée de couches multiples de l'histoire. À certains moments, le passé semble envahir - ou hanter - le présent, comme par exemple quand une photo d'un manifestant algérien blessé flotte comme un palimpseste spectral sur une image des touristes de nos jours devant un monument parisien. À d'autres moments par contre, les choix esthétiques de Lambert et Salva peuvent être dérangeants, particulièrement dans les cas des témoignages des victimes. Les témoignages sont filmés sous plusieurs angles, avec des coupes fréquentes d'un angle à un autre. L'objectif de ces coupes rapides et de la profusion d'images pourrait être de capter l'attention de jeunes spectateurs et de transmettre une grande quantité d'informations en peu de temps (le témoignage le plus long, celui de Benaissa, dure seulement six minutes et quarante-deux secondes), mais ces techniques pourraient également distraire certains spectateurs, et amoindrir la gravité des témoignages. Cependant, un aspect positif (voulu ou pas) de ce parti pris esthétique est de souligner la nature fragmentaire de chaque témoignage, et par moments la caméra respecte des moments de silence, avec des plans des mains ou des têtes penchées des témoins sans paroles.

Les témoignages personnels sont l'élément central de La Nuit oubliée, mais il est important de noter comment le webdocumentaire se situe comme une investigation qui s'élargit progressivement, comme l'enquête de l'Inspecteur Cadin dans Meurtres pour mémoire. La deuxième section, "En coulisses ", présente des entretiens avec des hommes politiques qui expliquent le contexte de l'époque. La troisième section, qui s'intitule « Un jour dans la Guerre d'Algérie ", fournit un peu plus d'éléments contextuels tout en démontrant un des écueils potentiels du webdocumentaire dans son état actuel. Le titre de la section est trompeur, puisqu'en réalité cette section présente sept moments-clés du conflit et sept massacres de civils entre 1945 et 1962, avec une brève description et des vidéos provenant des archives de l'INA. Malheureusement, en avril 2015, plusieurs des vidéos ne sont plus accessibles sur le site. Si des problèmes ou des changements technologiques rendent caducs des éléments du site web, et si le spectateur ne peut plus avoir accès aux informations, cela soulève un problème grave pour le webdocumentaire comme genre. Il est particulièrement regrettable que cette section ne fonctionne plus, car il est important de se souvenir que le 17 octobre n'était qu'un des événements dans une série de massacres qui sont souvent occultés dans la mémoire collective. Comme le note Rousso, " "le devoir de mémoire" et l'hypermnésie de certains épisodes de l'Histoire génèrent autant l'oubli que le souvenir ${ }^{31} »$.

24 Du côté droit de l'écran, il y a une carte de Paris navigable et un hyperlien nommé "Archives». La carte a trois onglets - «manif», "témoins», et «lieux»- qui permettent au spectateur de choisir entre trois versions de la carte et d'explorer de différentes couches d'histoire dans ce Paris virtuel. La carte "témoins" montre simplement les lieux de Paris où les témoins ont vu ce qu'ils racontent dans leurs témoignages, mais les cartes « manif » et « lieux » sont plus intéressantes. 


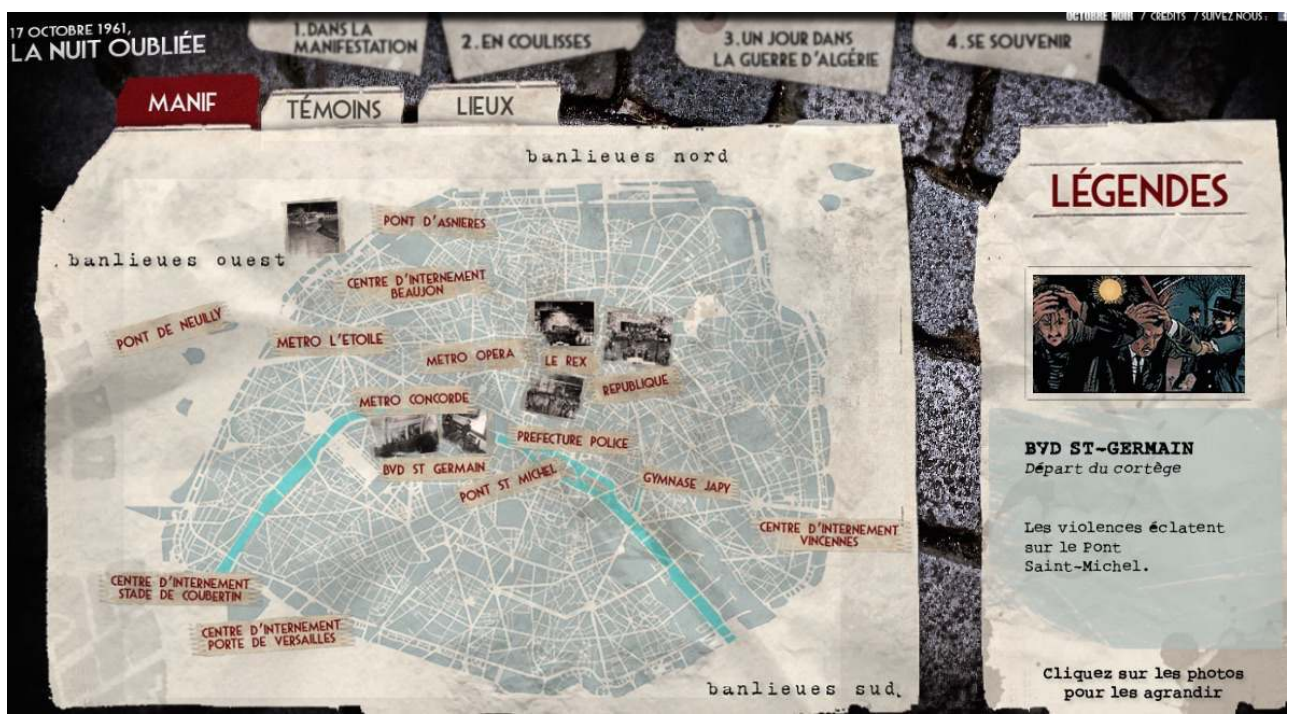

Figure 5 : La Nuit oubliée. Carte «manif ». (@ Olivier Lambert et Thomas Salva) noir, et la partie « lieux » visite certains des mêmes lieux aujourd'hui, à travers des photos de Thomas Salva, un des créateurs du webdocumentaire. Cette partie du site, qui porte la légende "Retourner sur les traces d'hier pour libérer la parole aujourd'hui », rappelle le parcours d'Amel et Omer dans La Seine était rouge ${ }^{32}$. Dans le roman, ils retracent le chemin de la manifestation, frappés par le contraste entre le présent et le passé, et ils essaient d'inscrire des traces de la violence policière occultée sur des monuments parisiens contemporains. Dans les photos de Salva, les images paisibles, voire banales, des rues parisiennes sont accompagnées par des légendes qui racontent la violence du 17 octobre et qui forcent le spectateur à réfléchir à ce qui s'est passé sur ces lieux en 1961, tout comme Amel et Omer créent «leur propre commémoration par l'entremise de graffiti rouges transgressifs ${ }^{33} »$.

Dans la partie "manif», la juxtaposition des images de bande dessinée et des photos d'archives ne pose pas de problème. Dans la partie « Archives ", en revanche, la frontière entre le réel et la fiction est beaucoup plus floue, avec des conséquences troublantes. Les archives sont une destination logique pour le webdocumentaire, à la fois dans sa structure narrative et aussi dans sa mission pédagogique. Il est important de donner des ressources pour ceux qui aimeraient apprendre davantage, mais il est essentiel d'indiquer ce qui appartient au domaine du réel, et ce qui appartient au domaine de la fiction. Dans les « archives » de La Nuit oubliée, les documents téléchargeables paraissent authentiques au premier coup d'œil, mais lorsqu'on les examine d'un peu plus près, il est évident que certains des documents ne le sont pas. Par exemple, dans le cas d'un document du «Registre de l'Institut médico-légal », avec une liste des morts, la même image d'une feuille de papier jaunie et froissée est reproduite sur chacune des sept pages, et en grossissant l'image, on voit que la liste des noms «tapée à la machine » est en fait superposée sur l'image de fond. Par exemple, on voit clairement que des caractères noirs sont superposés sur une agrafe dans plusieurs des images. Même si les représentations romanesques et cinématographiques ont joué un rôle essentiel en révélant les événements du 17 octobre, un tel mélange du réel et du fictif semble problématique pour une forme qui se veut webdocumentaire. Cette même tension réapparaît dans 17.10.61. 


\section{8 octobre LASMI Smaill \\ MEKLOUCHE Amar \\ 19 octobre ACHEMOUNE Lamara}

Figure 6 : La Nuit oubliée. Gros plan d'un document des «Archives». (@ Olivier Lambert et Thomas Salva)

\subsubsection{1}

Tandis que La Nuit oubliée se caractérise par son esthétique de bande dessinée, le webdocumentaire 17.10.61 créé par le collectif Raspouteam et produit par la société Agat Films \& Cie, est beaucoup plus sobre et cinématographique. Sur un fond noir, la page d'accueil présente une carte de Paris en gris que la Seine traverse comme un fleuve de sang. En haut de la page il y a huit photos de visages, et en cliquant sur les photos ou sur la carte, le spectateur peut visionner des témoignages filmés des personnages.

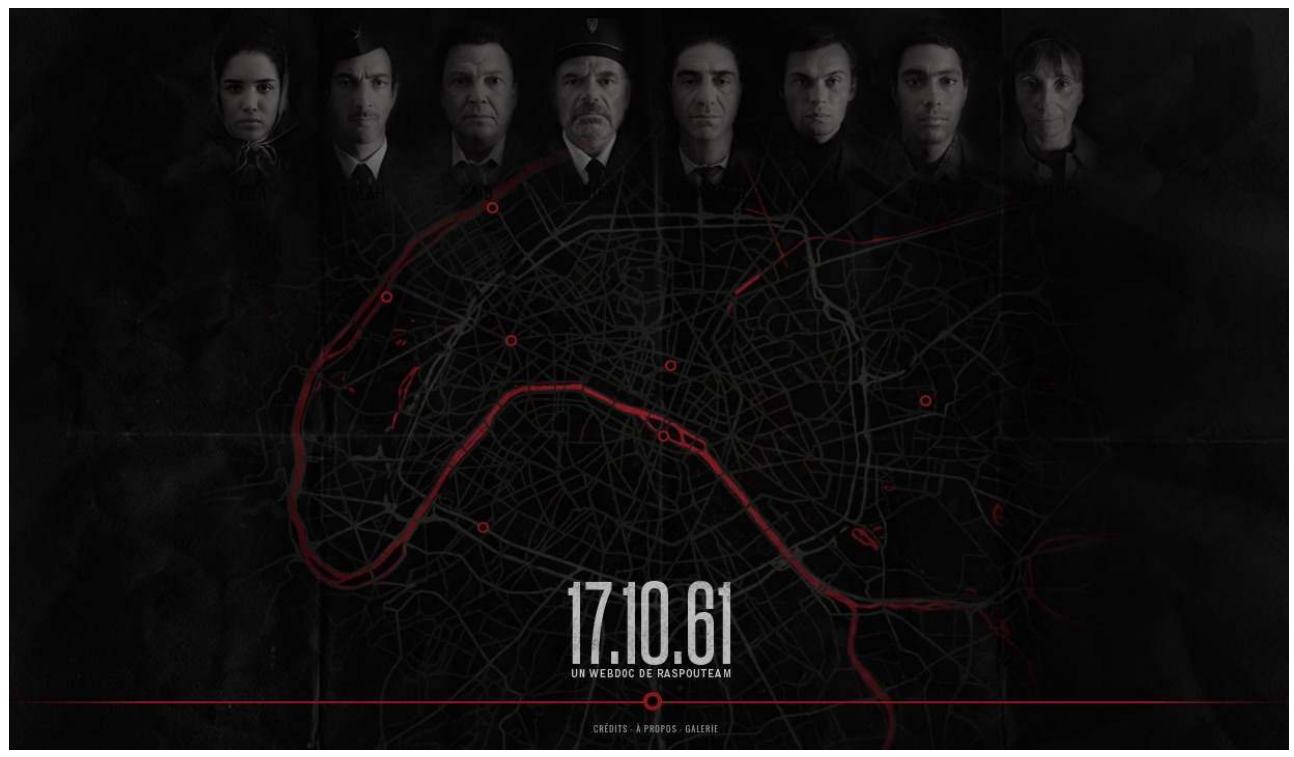

Figure 7 : 17.10.61. Page d'accueil. (@ Raspouteam)

La différence majeure entre La Nuit oubliée et 17.10.61 réside dans le statut de ces témoignages, car tandis que La Nuit oubliée présente des témoins réels, 17.10.61 présente des témoignages composites romancés. Les visages sur le site sont ceux des comédiens professionnels (dont deux lauréats du César) qui jouent des rôles de témoins. Même si les 
créateurs affirment que "Tous les personnages de ces films sont inspirés de vrais témoignages» dans la courte séquence qui ouvre le webdocumentaire, les « témoignages » présentés sur le site frappent moins fort que les vrais témoignages de $L a$ Nuit oubliée ${ }^{34}$. Si le statut fictif des attestations amoindrit la gravité des témoignages, il faut noter que le webdocumentaire est d'une beauté et d'une cohérence esthétique qui le différencie de la Nuit oubliée. Qui plus est, les créateurs font plus confiance au spectateur, et la navigation dans le site est plus fluide, plus ouverte. Dans La Nuit oubliée, il y a un parcours à suivre, et le spectateur est guidé par des personnages d'Octobre noir, tandis que dans 17.10.61, le spectateur est libre de déambuler à sa guise autour de Paris.

L'esthétique des séquences des témoignages est très similaire à La Nuit oubliée: un amalgame de photographies et de séquences provenant des archives et aussi de nos jours. L'usage de la surimpression, comme dans une séquence où des manifestants algériens apparaissent lentement et puis disparaissent du cadre rappelle l'effet esthétique employé par Amel et Omer dans La Seine était rouge, où selon Anne Donadey, ils créent « un palimpseste historique mettant en lumière de manière subversive à la fois les événements de la guerre d'Algérie et le manque de commémoration officielle à ce sujet en France ${ }^{35}$ ».

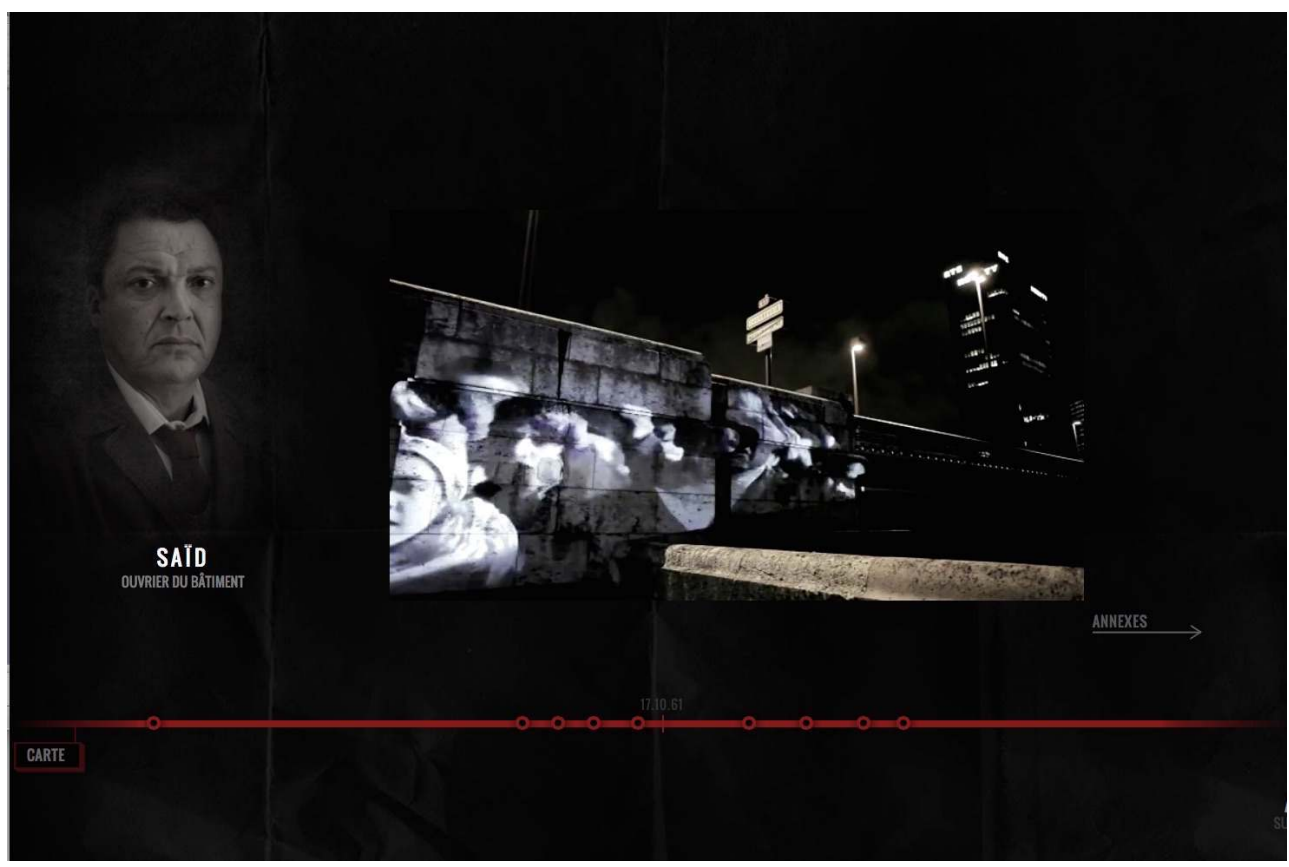

Figure 8 : 17.10.61. Témoignage de « Saïd ». (@ Raspouteam)

Comme La Seine était rouge, 17.10.61 offre une variété de témoignages, passant d'un harki à un policier à une porteuse de valise. Le choix de créer des témoins fictifs permet aux créateurs d'inclure une gamme diverse de perspectives, et chaque histoire est suivie par de courts entretiens filmés avec des historiens qui fournissent le contexte historique pour les différents sujets abordés dans les récits des acteurs. Une bibliographie et des matériaux d'archives accompagnent chaque témoignage, et de cette manière, la structure narrative ressemble à celle de La Nuit oubliée, passant du témoignage au contexte historique, suivi par des suggestions pour des recherches plus approfondies. Le budget et la production professionnelle rendent les séquences du webdocumentaire très convaincantes, mais cela présente aussi un danger très réel quand les vidéos sont soustraites au contexte pédagogique du webdocumentaire. Les différentes séquences du webdocumentaire sont hébergées sur le site Vimeo, et elles sont facilement téléchargées 
et disséminées par n'importe qui. Par exemple, sur YouTube, un utilisateur a mis en ligne une des séquences de 17.10.61, sans aucune indication quand au statut fictif du témoignage 36 .

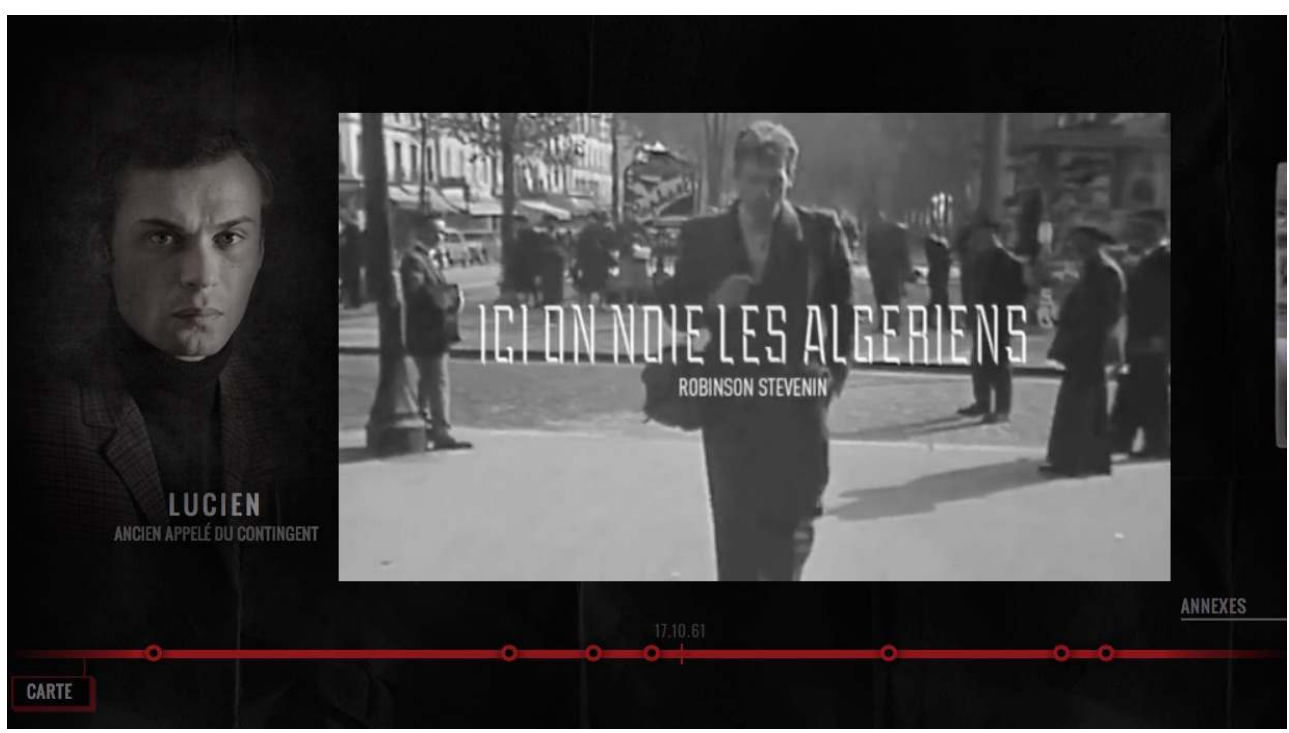

Figure 9 : 17.10.61. Témoignage de «Lucien». (@ Raspouteam)

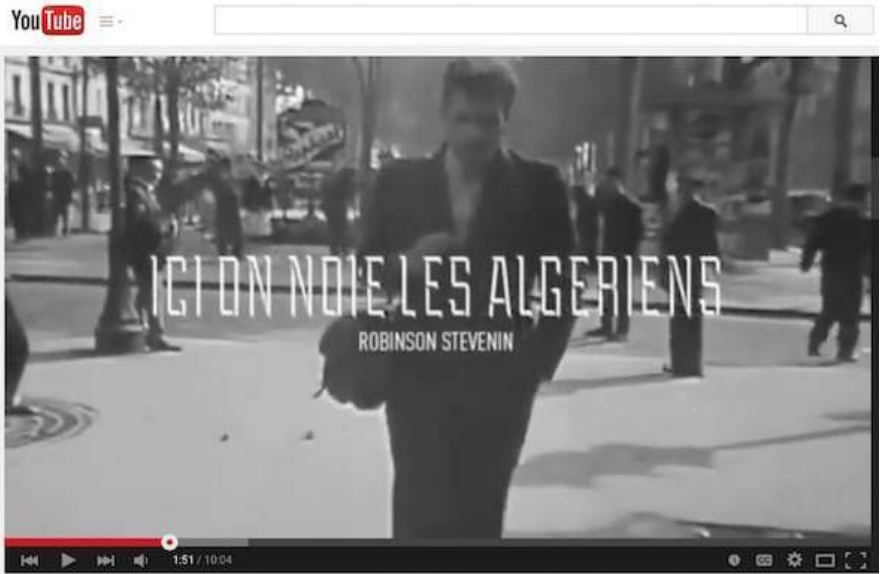

tous les français ne sont pas raciste BEAUCOUP SONT VS LA GUERRE LE TEMOIN EST UN FR

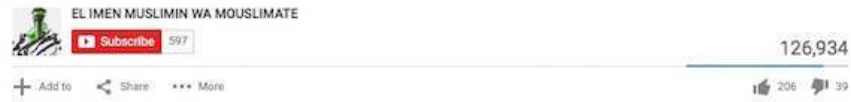

$+\cos 10<\sin 2 \ldots \operatorname{son}$

1306

Published on Nov 2, 2012

Un ancien soldart tancelie temoiGNe

ALL COMMENTS (163)

Share your thought

Figure 10 : YouTube. Témoignage de « Lucien». (@ Raspouteam)

31 La séquence, qui raconte un viol et des violences commises par des soldats français en Algérie avant de passer aux événements du 17 octobre, est présentée comme un vrai témoignage du soldat "Lucien ", malgré le fait que le nom de l'acteur Robinson Stevenin apparait sur l'écran. En avril 2015, la vidéo a déjà été visionnée plus de 126000 fois et il y a plus de 160 commentaires, pour la plupart très virulents. C'est un rappel urgent et sombre des enjeux de la situation, et cela démontre aussi la manière dont même des 
instruments pédagogiques peuvent être détournés et exploités dans les conflits de mémoire.

L'appel au devoir de mémoire devient un lieu commun contemporain, ainsi que l'idée que le récit historique se justifie par le fait de ne pas répéter les leçons du passé. Mais c'est un appel très creux et sans effet si on ne l'articule pas à un discours historique précis. Du coup, l'avantage de la forme webdocumentaire est aussi, dans cette perspective pédagogique, un inconvénient. Si la fragmentation du propos et la non-linéarité permettent de renouveler les formes de la diction contemporaine de l'histoire multidirectionnelle, de les adapter à la forme web et à un public qui ne lit plus de livres, elles sont aussi un obstacle peut-être à la formulation d'un propos cohérent qui dénoue les nœuds de l'histoire.

\section{NOTES}

1. Éric Conan et Henry Rousso, Vichy, un passé qui ne passe pas, Paris, Fayard, 1994.

2. La Nuit oubliée, <http://www.lemonde.fr/societe/visuel/2011/10/17/la-nuitoubliee_1587567_3224.html>

3. 19.10.1961, <http://raspouteam.org/1961/home.html>

4. Henry Rousso, La Dernière catastrophe, Paris, Gallimard, 2012, p. 12.

5. Didier Daeninckx, Meurtres pour mémoire, Paris, Gallimard, 1984.

6. Leïla Sebbar, La Seine était rouge : Paris, octobre 1961, Paris, Thierry Magnier, 1999.

7. Michael Rothberg, «Introduction : From Memory to Memory », Yale French Studies, vol. 118/119, New Haven, 2010, p. 7.

8. Henry Rousso, «les Raisins verts de la guerre d'Algérie », dans La Guerre d'Algérie (1954-1962), Paris, Odile Jacob, 2005, p. 141.

9. Michel Laronde, " "Effets d'Histoire": Représenter l'Histoire coloniale forclose ", International Journal of Francophone Studies, n¹0, 2007, p. 145.

10. Henry Rousso, « les Raisins verts de la guerre d'Algérie », op. cit., p. 142.

11. « mémoire multidirectionnelle »

12. Michael Rothberg, Multidirectional Memory : Remembering the Holocaust in the Age of Decolonization, Stanford, Stanford University Press, 2007, p. 4.

13. Henry Rousso, « les Raisins verts de la guerre d'Algérie », op. cit., p. 143-144.

14. Annette Wievorka, L'Ère du témoin, Paris, Plon, 1998, p. 14.

15. Henry Rousso, « les Raisins verts de la guerre d'Algérie », op. cit., p. 143.

16. Michael Rothberg, Multidirectional Memory : Remembering the Holocaust in the Age of Decolonization, op. cit., p. 234, 236. «Le roman relie un polar construit sur le mystère, la détection, et la révélation avec une intrigue basée sur la transmission historique intergénérationnelle. [...] Le but de Meurtre - le mystère qu'il crée pour résoudre -est le lien entre différentes ères et la persistance d'un passé irrésolu dans le présent ». 
17. Claire Gorarra, «Tracking Down the Past: The Detective as Historian in Texts by Patrick Modiano and Didier Daeninckx ", dans Anne Mullen and Emer O’Beirne (eds.), Crime Scenes : Detective Narratives in European Culture Since 1945, Amsterdam, Rodopi, 2000, p. 287.

18. Anne Donadey, "Retour sur mémoire : La Seine était rouge de Leilla Sebbar ", dans Michel Laronde (ed.), Leila Sebbar, Paris, L'Harmattan, 2003, p. 192.

19. Anne Donadey, ibid., p. 192.

20. Jonathan Lewis, «Filling in the Blanks: Memories of 17 October 1961 in Leila Sebbar's La Seine était rouge ", Modern \& Contemporary France, vol. 20, no 3, 2012, p. 314-315. «Sebbar ne présente pas au lecteur une version singulière et monolithique de l'histoire pour résoudre le manque de transmission historique en ce qui concerne le 17 octobre 1961 et la Guerre d'Algérie. Au contraire, le récit qui en résulte est inévitablement fragmenté, composé d'informations fragmentaires, recueillies de différentes sources, et le récit change constamment entre la quête d'Amel de retracer le chemin des manifestants algériens en compagnie d'Omer et la description des clips du film de Louis ».

21. Didier Daeninckx and Mako, Octobre noir, Paris, Ad Libris, 2011.

22. La Nuit oubliée, op. cit.

23. Benjamin Stora, « Préface », Octobre noir, op. cit., p. 12.

24. Annette Wieviorka, L'Ère du témoin, op. cit., p. 98.

25. Annette Wieviorka, ibid., p. 96-97.

26. La Nuit oubliée, op. cit.

27. Michael Rothberg, Multidirectional Memory: Remembering the Holocaust in the Age of Decolonization, op. cit., p. 279. «En conséquence du hasard de la naissance, des sujets nationaux (et autres) héritent l'impératif d'enquêter sur les multiples formes de violence qui ont été commises en leur nom ».

28. Philippe Bernard and Christine Garin, "Archives du 'Monde' (17 octobre 2001) - Le massacre du 17 octobre 1961 obtient un début de reconnaissance officielle", Le Monde, 17 October 2011, <http://www.lemonde.fr/societe/article/2011/10/17/archives-dumonde-17-octobre-2001-le-massacre-du-17-octobre-1961-obtient-un-debut-dereconnaissance-officielle_1588198_3224.html>.

29. Azenstarck est un des témoins qui apparaît dans le webdocumentaire.

30. L'Institut National de l'Audiovisuel et la Bibliothèque de Documentation Internationale Contemporaine - Musee d'Histoire Contemporaine.

31. Henry Rousso, « les Raisins verts de la guerre d'Algérie », op. cit., p. 143.

32. La Nuit oubliée, op. cit.

33. Anne Donadey, « Retour sur mémoire : La Seine était rouge de Leïla Sebbar », op. cit., p. 195.

34. 17.10.61, op. cit.

35. Anne Donadey, ibid., p. 195.

36. <http://www.youtube.com/watch?v=7QEasP7YziI\&list=PLD2eGZp5_B-

EHI5JDOctRE8b9DRihyQT5>. 


\section{ABSTRACTS}

The legacy of the Algerian War has become the latest " passé qui ne passe pas » to borrow an oftquoted expression from the title of Éric Conan and Henry Rousso's study of the Vichy era. Polemics surrounding the commemoration of the fiftieth anniversary of the end of the war demonstrate the extent to which that legacy remains deeply unsettled and unsettling to many. One particular event - the police massacre of Algerian protesters in Paris on 17 October, $1961-$ has come to stand as a metonymy for the worst excesses of the period. This chapter examines the aesthetic and narrative strategies deployed in two web-documentaries - La Nuit oubliée and 17.10.61 - that were published in 2011 to commemorate the fiftieth anniversary of the events. These interactive multimedia websites raise a number of intriguing issues as they strive to find a new way in which to represent relatively recent historical events, navigating between what Henry Rousso describes as « la tension, parfois l'opposition entre l'histoire et la mémoire, entre la connaissance et l'expérience, entre la distance et la proximité, entre l'objectivité et la subjectivité, entre le chercheur et le témoin ».

L'héritage de la Guerre d'Algérie est devenu le plus récent “passé qui ne passe pas" pour emprunter le titre célèbre de l'étude des années Vichy écrite par Éric Conan et Henry Rousso. Plusieurs controverses ont éclaté en France autour de la commémoration du cinquantième anniversaire de la fin de la guerre, montrant jusqu'à quel point cette histoire reste irrésolue et perturbante. Un événement en particulier - le massacre des manifestants algériens à Paris le 17 octobre 1961 - est devenu une métonymie pour les pires excès de l'époque. En 2011, lors du cinquantième anniversaire de ces événements, deux webdocumentaires - La Nuit oubliée et 17.10.61 - ont paru sur Internet avec le but d'éclaircir ce moment historique pour une nouvelle génération de jeunes. Cet article analyse les stratégies esthétiques et narratives employées par ces deux webdocumentaires dans leur quête de nouvelles manières de représenter une histoire longtemps cachée, naviguant entre ce que Henry Rousso décrit dans La Dernière catastrophe comme, « la tension, parfois l'opposition entre l'histoire et la mémoire, entre la connaissance et l'expérience, entre la distance et la proximité, entre l'objectivité et la subjectivité, entre le chercheur et le témoin ».

\section{AUTHOR}

\section{PHILIPPE BRAND}

Assistant Professor de littérature française à Lewis \& Clark College (Portland, Oregon, ÉtatsUnis). Ses recherches se concentrent sur la littérature française contemporaine, en particulier la littérature et l'histoire, les fictions post-apocalyptiques, et le retour au récit depuis les années 1980. Il a publié des articles dans The French Review, Contemporary French and Francophone Studies, et la Revue Critique de Fixxion Française Contemporaine. 